\title{
The Variation of the Manifold Under the Time
}

\author{
M. EL-Ghoul \\ Mathematics department, Faculty of Science \\ Tanta University, Tanta, Egypt \\ E-mail: m.elghoul@msn.com \\ H. Ahmed \\ Mathematics department, Faculty of Girl \\ Ain shams University, Egypt \\ E-mail: hebanoha2005@yahoo.com
}

Received: November 30, 2010 Accepted: December 24, 2010 doi:10.5539/jmr.v3n2p119

\begin{abstract}
In this papre, we introduce the variation of the manifold under the time, the variation in the volume, the variation in the dimension and the variation in the position are discussed.
\end{abstract}

AMS Subject Classification 2000: 51H10, 57N10.

Keyword: Dynamical manifold

\section{Definitions and Background}

1- Dynamical system: The notation of a dynamical system is the mathematical formalization of the general scientific concept of a deterministic process.

The evolution of a dynamical system means change in the state of the system with time $t \in \mathrm{T}$ where $\mathrm{T}$ is an ordered set.We will consider two types of dynamical systems.

Some with continuous time $\mathrm{T}=\mathrm{R}$, and another with discrete(integer) time $\mathrm{T}=\mathrm{Z}$.

System of the first type are called continuous-time dynamical systems, the second are termed discrete-time dynamical system.[M.M.A.Ramadan, 2008].

2- n-manifolds: The $n$-dimensional manifold is a Housdorff space (Space stisfies the $T_{2}$ separation axiom) such that each point has an open neighborhood homeomorphic to the open n- dimensional disc.

$\mathrm{U}^{n}=\left\{x \in R^{n}:\|x\|<1\right\}$.

Where $\mathrm{n}$ is positive integer.[Massey W.S, 1973].

3- Housdorff space: A topolgical space X which satisfy the following axiom "Every two distinct point of X have disjoint neighborhoods in X"..[Massey W.S, 1973].

\section{The main results}

Aiming to our study, we will introduce the variation of manifold under the time.

2.1 The variation in the volume

2.1.1 The variaton in the volume and the shape not variation

See Fig.(1) form the 1-manifold $L$ after some time it will be $L^{\backslash}, L^{\backslash}$ is 1-manifold (The same shape of L).

The limit in this case $\left(\mathrm{L}^{\backslash}\right)$ goes to $\mathrm{R}^{1}$.

See Fig.(2) form the 2-manifold c after some time it will be $\mathrm{c}^{\backslash}, \mathrm{c} \backslash$ is 2-manifold (The same shape of c).

The limit in this case $\left(c^{\backslash}\right)$ goes to $\mathrm{R}^{2}$.

See Fig.(3) form the 3-manifold $b$ after some time it will be $b^{\backslash}, b^{\backslash}$ is 3-manifold (The same shape of $b$ ).

The limit in this case $\left(b^{\backslash}\right)$ goes to $\mathrm{R}^{3}$.

2.1.2 The variation in the volume and the shape variation

See Fig.(4) form the 1-manifold $\mathrm{L}$ after some trime it will be $\mathrm{L} \backslash, \mathrm{L} \backslash$ is 1 -manifold (The shape variant).

The limit in this case $\left(\mathrm{L}^{\backslash}\right)$ goes to $\mathrm{R}^{1}$. 
See Fig.(5) form the 2-manifold c after some time it will be $\mathrm{c}^{\backslash}, \mathrm{c} \backslash$ is 2-manifold (The shape variant).

The limit in this case $\left(\mathrm{c}^{`}\right)$ goes to $\mathrm{R}^{2}$.

See Fig.(6) form the 3-manifold $b$ after some time it will be $b^{\backslash}, b^{\backslash}$ is 3-manifold (The shape variant).

The limit in this case $\left(b^{\backslash}\right)$ goes to $R^{3}$.

\subsection{The variation in the dimension}

2.2.1 From 0-dimension to 1-dimension

See Fig.(7) form the 0-manifold a after some time it will be $\mathrm{L}, \mathrm{L}$ is 1-dimensiona.

The limit in this case $(\mathrm{L})$ gose to $\mathrm{R}^{1}$.

2.2.2 From 1 -dimension to 2 - dimension

See Fig.(8) form 1-manifold $\mathrm{L}$ after some time it will be $\mathrm{c}, \mathrm{c}$ is 2-dimension.

The limit in this case(c) goes to $\mathrm{R}^{2}$.

2.2.3 From 0 - dimension to 2 - dimension

See Fig.(9) form the 0-manifold a after some time it will be c ,c is 2-dimension.

The limit in the case(c) goes to $\mathrm{R}^{2}$.

This the variation is ungradually.

2.3 The variation in the position

2.3.1 The variation of 1- manifold

See Fig.(10) form a point to straight line.

\subsubsection{The variation of 2 - manifold}

See Fig.(11) form a point to volume.

See Fig.(12) form a point to solid.

2.4 If there are two manifolds and the two manifolds are identical

See Fig.(13) form the 1-manifold $L$ after some time it will be $L^{\prime \prime \prime}, L^{\prime \backslash}$ is 1 -manifold (The outside manifold and the inside manifold are identical).

The limit in this case $\left(\mathrm{L}^{11}\right)$ goes to $\mathrm{R}^{1}$.

\subsection{There are many 2-manifold inside the manifold}

See Fig.(14) refer to the inside manifolds increasing, and the limit of the inside manifold goes to $\mathrm{R}^{2}$.

See Fig.(15) refer to the inside manifolds decreasing, and the limit of the inside manifold goes to $\mathrm{R}^{2}$.

2.6 There are many 3-manifold inside the manifold

See Fig.(16) refer to the inside manifolds increasing, and the limit of the inside manifold goes to $\mathrm{R}^{3}$.

See Fig.(17) refer to the inside manifolds decreasing, and the limit of the inside manifold goes to $\mathrm{R}^{3}$.

2.7 The variation of the manifold with boundary under the time

See Fig.(18) The limit in this case goes to $\mathrm{R}^{3}$.

See Fig.(19) The limit in this case goes to $\mathrm{R}^{2}$.

\section{Theorem 1:}

The variation of the manifold of dimension $\mathrm{n}$ by the time into another $\mathrm{n}$-manifold is in a volume, curvature and torsion.

Proof:

See Fig(20).

Let $\mathrm{M}$ be a manifold of dimension $\mathrm{n}, \mathrm{L}$ arc length, $\mathrm{K}$ is the curvature, $\tau$ is a torsion, then:

$\mathrm{L}=\int|I| d t, d x \cdot d x=I$

Where $\mathrm{I}$ is the first fundamental form.

Since $x \underset{t_{1}}{\rightarrow} x^{\backslash}$ 
Then $\underset{\rightarrow}{t_{1}} \mathrm{~L}^{\backslash}=\int\left|I^{\backslash}\right| d t$

Also $t=k n, t=\left(\frac{d x}{d t}\right)^{\cdot t_{1}} \rightarrow\left(\frac{d x}{d t}\right)$.

$b=\tau n, \mathrm{~b}=\mathrm{t} \wedge \mathrm{n} \underset{t_{1}}{\rightarrow} \mathrm{t} \backslash \wedge \mathrm{n}^{\backslash}=b^{\backslash}$.

\section{Lemma 1:}

The variation in a manifold of dimension $\mathrm{n}$ without boundary is a manifold with boundaries.

\section{Proof:}

See Fig.(21).

Let $\mathrm{G}: \stackrel{\mathrm{M}}{t_{1}} \mathrm{M}^{\backslash}$

Where $\mathrm{M}$ is a manifold without boundary, and $\mathbf{M}^{\backslash}$ is a manifold with boundary.

\section{Lemma 2:}

The converse of the above lemma is true.

\section{Proof:}

See Fig.(22).

Let $\mathrm{G}: \mathrm{M} \rightarrow \mathrm{M}^{\backslash}$

Where $\mathrm{M}$ is a manifold with boundary, and $\mathbf{M}^{\backslash}$ is a manifold with out boundary.

\section{Applications:}

1-Growth of seeds of plants.

2-A leaf is sick and the sickness is spreaded in every side of it then it will form a net.

\section{References}

P.J.Giblin. (1977). Graphs, Surfaces and Homology, An Introduction to Algebraic Topology, 11 New Fetter Lane, London EC4P4EE.

Massey W.S. (1973). Algebraic Topology, An Introduction.Harcourt, Brace\& World, Inc, NewYork U.S.A.

Arthur T. White. (1973). Graphs, Group and Surface. Western Michigan University Kalamazoo, Mich.U.S.A.

Manfredo P. do Carmo. (1976). Differential Geomerty of Curves and Surfaces.Instituto de Matemetica Pura e Aplicade(IMPA) Riode Janeiro,Brazil.

M.M.A.Ramdan. (2008). A study on graphs and knots, Ph.D.Thesis.Univ.Minufiya.Egypt.

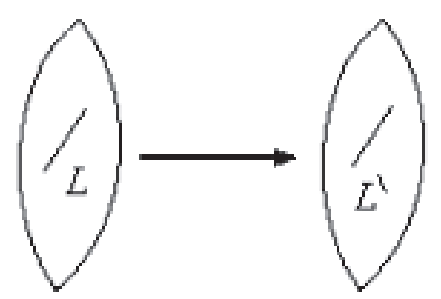

Figure 1

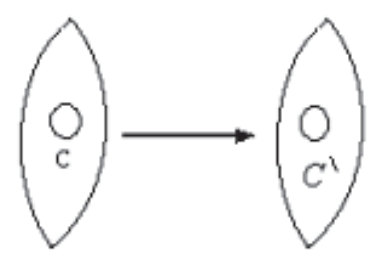

Figure 2 


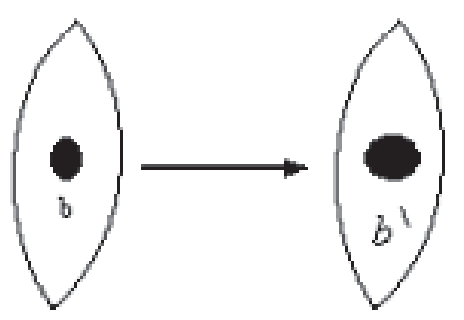

Figure 3

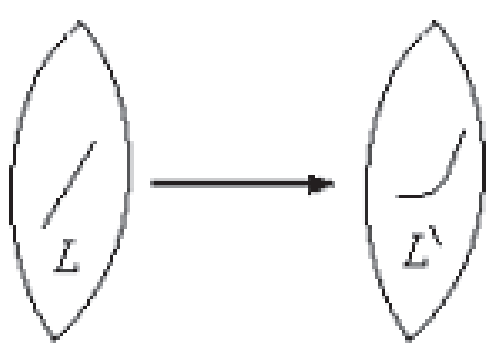

Figure 4

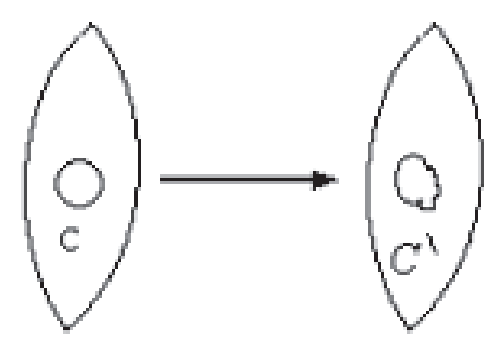

Figure 5

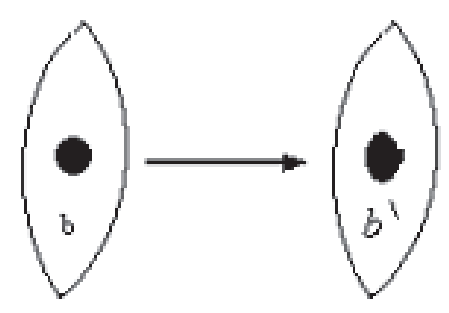

Figure 6

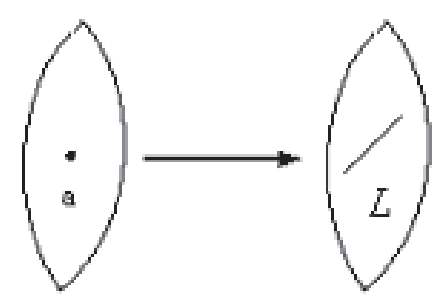


Figure 7

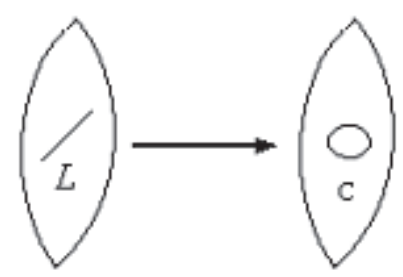

Figure 8

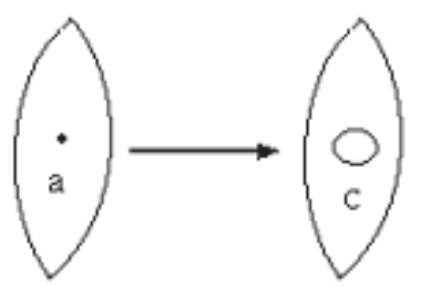

Figure 9

a

a

b

Figure 10

a

Figure 11

$\dot{a}$

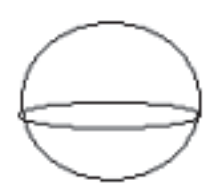

Figure 12 


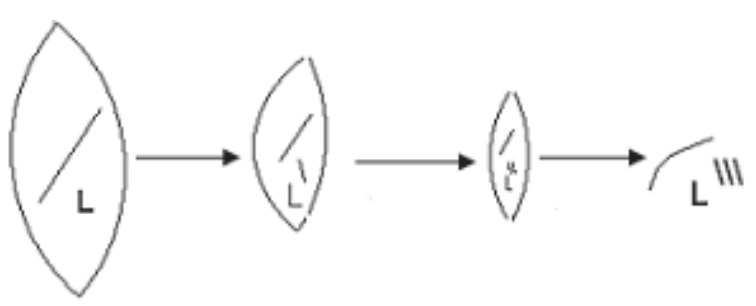

Figure 13

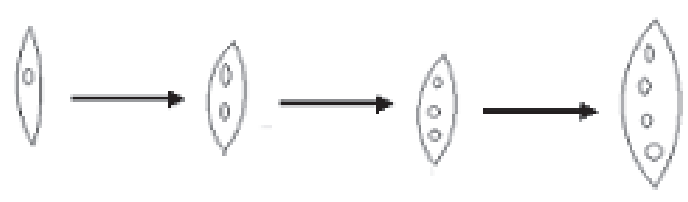

Figure 14

$$
\left(\begin{array}{l}
0 \\
0
\end{array}\right) \rightarrow\left(\begin{array}{l}
0 \\
0
\end{array}\right) \rightarrow 0
$$

Figure 15

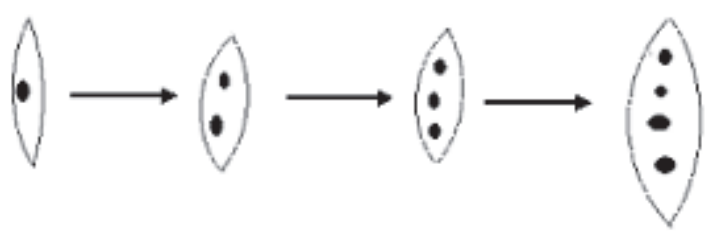

Figure 16

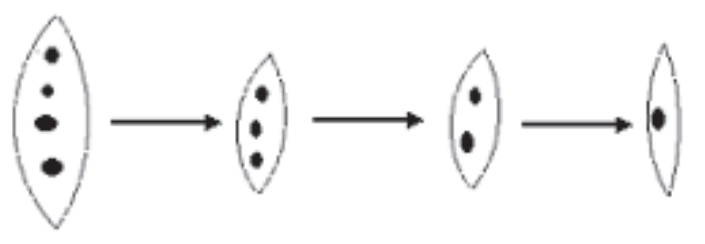

Figure 17

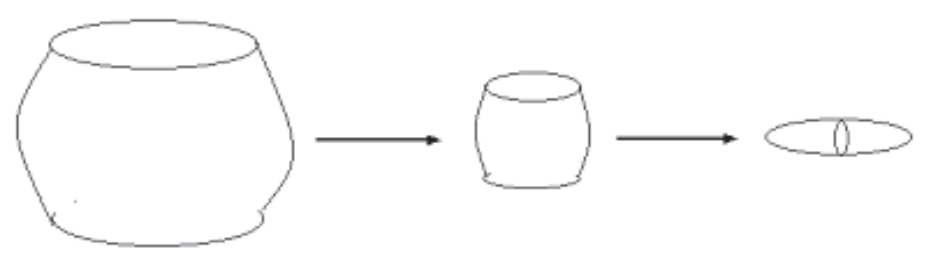

Figure 18 


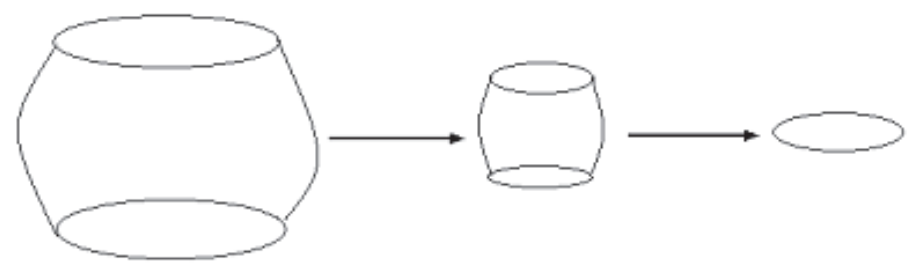

Figure 19

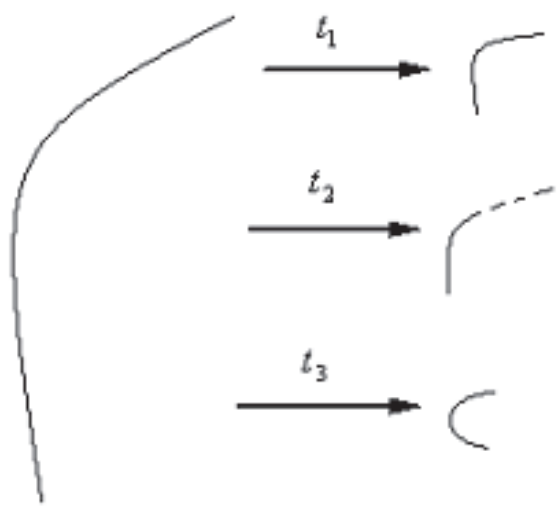

Figure 20

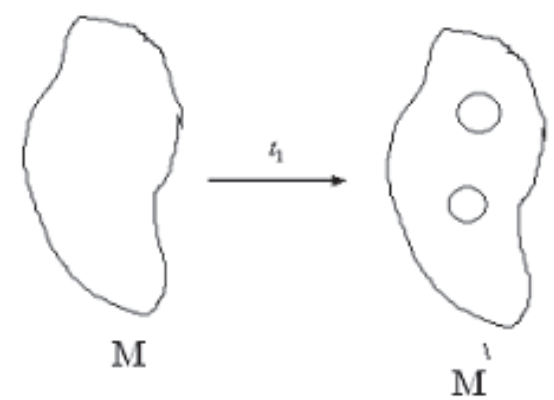

Figure 21

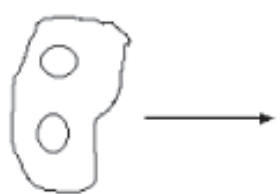

M

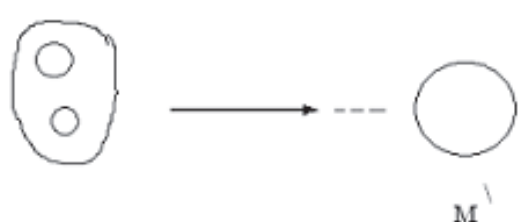

M

Figure 22 